\title{
Autoantibody Clustering in Systemic Lupus Erythematosus-Associated Pulmonary Arterial Hypertension
}

\author{
Ritasman Baisya ${ }^{1}$ Phani Kumar Devarasetti ${ }^{1}$ Murthy G S R ${ }^{2} \quad$ Liza Rajasekhar $^{1}$ \\ 1Department of Clinical Immunology and Rheumatology, Nizam's \\ Institute of Medical Sciences, Hyderabad, Telangana, India \\ 2Statistical Quality Control and Operational Research Division, \\ Indian Statistical Institute, Kolkata, West Bengal, India62100105 \\ Address for correspondence Ritasman Baisya, MD, Department \\ of Clinical Immunology and Rheumatology, Nizam's Institute \\ of Medical Sciences, Hyderabad, Telangana 700118, India \\ (e-mail: ritasman91@gmail.com).
}

Ind J Car Dis Wom 2021;6:100-105.

\begin{abstract}
Keywords

- SLE-PAH

- clustering analysis

- autoantibody

Systemic lupus erythematous-associated pulmonary arterial hypertension (SLE-PAH) is one of the important causes of mortality in lupus patients. Different autoantibodies are associated with SLE-PAH which can predict its future development. The objective of the study was to identify distinct autoantibody-based clusters in SLE-PAH patients and to compare demographic characters, clinical phenotypes, and therapeutic strategy across the clusters. Three distinct autoantibody clusters were identified using k-means cluster analysis in 71 SLE-PAH patients. Cluster 1 had predominant Sm-RNP, Smith, SS-A association; cluster 2 had no definite autoantibody association; and cluster 3 was associated with nucleosome, histone, dsDNA, and ribosomal $\mathrm{P}$ protein. Patients in cluster 3 had a highly active disease while those in cluster 1 had significant cytopenia. Mean age and mean right ventricular systolic pressure (RVSP) were both high in cluster 2 , indicating later-onset PAH in this group. This was the first autoantibody-based cluster analysis study in SLE-PAH patients in India which confirmed that autoantibodies did exist as clusters and the presence of definite autoantibodies can predict future development of pulmonary hypertension in these patients.
\end{abstract}

\section{Abstract Image}

DOI https://doi.org/ $10.1055 / \mathrm{s}-0041-1732510$

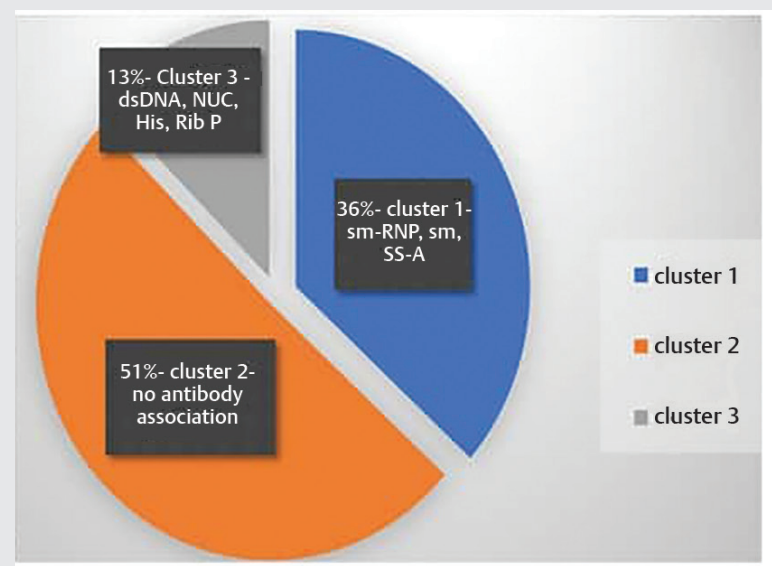

\begin{tabular}{|c|c|}
\hline & $\begin{array}{l}\text { Cluster } 3 \text { patients had } \\
\text { highly active disease }\end{array}$ \\
\hline & $\begin{array}{l}\text { Cluster } 1 \text { had significant } \\
\text { cytopenia }\end{array}$ \\
\hline • & $\begin{array}{l}\text { Cluster } 2 \text { - mean age and } \\
\text { mean right ventricular } \\
\text { systolic pressure were high } \\
\text { indicating later onset PAH }\end{array}$ \\
\hline
\end{tabular}

(c) 2021. Women in Cardiology and Related Sciences.

This is an open access article published by Thieme under the terms of the Creative Commons Attribution-NonDerivative-NonCommercial-License, permitting copying and reproduction so long as the original work is given appropriate credit. Contents may not be used for commercial purposes, or adapted, remixed, transformed or built upon. (https://creativecommons.org/licenses/by-nc-nd/4.0/).

Thieme Medical and Scientific Publishers Pvt. Ltd. A-12, 2nd Floor, Sector 2, Noida-201301 UP, India 


\section{Introduction}

Pulmonary arterial hypertension (PAH) is a potential complication of connective tissue diseases like scleroderma, systemic lupus erythematosus (SLE), and mixed connective tissue disorder (MCTD). It is one of the causes of death in SLE patients. Due to the heterogeneity and complexity of the underlying disease, pathogenesis, prevalence, risk factors, and the natural history of PAH in SLE remain poorly understood. ${ }^{1}$ As a result, there are no definite guidelines for screening, managing, and monitoring pulmonary hypertension in SLE patients.

Several autoantibodies are associated with PAH in SLE patients. Associations with antiribonucleoprotein $\left(\right.$ anti-RNP) ${ }^{2}$ and anticardiolipin antibodies ${ }^{3}$ have been reported in Chinese SLE cohorts. At the same time, Prabu et al ${ }^{4}$ found an association of PAH with lupus anticoagulant in an SLE cohort from the United Kingdom. Anti-Ro and anti-La have been associated with PAH in a French SLE cohort. ${ }^{5}$ Till now, the literature on antibody association in Indian lupus PAH patients is limited. A recently published study from the Hopkins Lupus Cohort where lupus phenotype associated with PAH was defined using multiple autoantibodies. ${ }^{1}$ Here clustering analysis was used for the first time to define five autoantibody clusters in lupus-PAH. In the present study, clustering analysis has been used to test the hypothesis that this technique would bring out unique phenotypic groups of PAH in Indian lupus patients. This method can help drive future investigations into risk factors and mediators of disease, enabling better targeted therapeutic approaches.

In another study by Sun et $\mathrm{al}^{6}{ }^{6}$ two distinct phenotypic clusters of SLE-PAH were identified based on disease activity. They identified two clusters-the one with high disease activity had serositis, nephritis, rash, and other disease manifestations, while the one with low disease activity had the purer form of PAH. Our study has combined autoantibodies with disease activity to generate distinct phenotypes in Indian lupus PAH patients.

\section{Objectives}

- Our study had the following objectives:

- To identify distinct autoantibody-based clusters in SLE-PAH patients using cluster analysis.

- To compare demographic characters, clinical phenotypes, and therapeutic strategies across the clusters.

\section{Methodology \\ Patients}

It was an institution-based observational study carried out in the Department of Rheumatology at Nizam's Institute of Medical Sciences (NIMS), a tertiary care center in South India. Seventy-one SLE patients with the diagnosis of PAH as per definition criteria were taken as the case. Thirty-five of these patients were part of a large inception cohort named INSPIRE, conducted throughout India. The rest of the population was from the patients admitted or presented in the outpatient department (OPD) and ward in rheumatology. Data were collected for 3 months from August 2020 to October 2020. The institutional ethics committee approved the study, and written informed consent was obtained from all patients, including separate consent forms for minor patients before participation.

\section{Inclusion Criteria}

Following were the inclusion criteria for the study:

- SLE patients, both juvenile and adult, fulfilling the "SLICC 2012" (SLE International Collaboration Clinics [SLICC] classification criteria) or ACR-EULAR 2019 (American College of Rheumatology - European League Against Rheumatism) classification criteria evaluated as outpatient or admitted as inpatient with PAH as a cardiopulmonary complication were included as study participants.

- PAH was defined as a right ventricular systolic pressure (RVSP) of greater than or equal to $40 \mathrm{~mm} \mathrm{Hg}$ on transthoracic echocardiogram and clinical symptoms of dyspnea-loud P2 on auscultation-and other clinical signs suggestive of pulmonary hypertension.

\section{Exclusion Criteria}

- Following were the exclusion criteria for the study: Previously diagnosed cardiac disease, like rheumatic heart disease (RHD) and congenital heart disease, before lupus onset.

- Pregnant and lactating patients.

\section{Study Parameters}

The study parameters were as follows:

- Demographic variables: Variables including age, sex, duration of disease, and duration of PAH were recorded.

- Clinical history: Detailed history of cutaneous findings (skin thickening, Raynaud phenomenon, vasculitic rash, and sicca symptoms), neuropsychiatric (cerebro-vascular accident [CVA], mononeuritis multiplex [MNM], SLE disease Activity Index [SLEDAI]), musculoskeletal (myositis), renal, hematologic, pregnancy morbidity, interstitial lung disease, serositis were recorded.

- Treatment history:Detailed history of steroids, immunosuppressive medication (cyclophosphamide), PAH medication (PDE 5 inhibitor-sildenafil/tadalafil-and endothelin receptor antagonist-ambrisentan) were taken.

- Disease activity:SLEDAI was calculated for all the patients. Those with SLEDAI $\geq 6$ were considered high disease activity, and those with SLEDAI $<6$ with no evidence of hemolytic anemia, thrombocytopenia, or major organ involvement were considered low disease activity groups.

\section{Laboratory Tests}

The following laboratory tests were done:

- Two-dimensional (2D) echocardiographic analysis: Mean RVSP (in mm Hg) was calculated for all of the study population, and severity of PAH was based on the mean value of RVSP. More than 40 to $49 \mathrm{~mm} \mathrm{Hg}$-mild PAH; 50 to $60 \mathrm{~mm} \mathrm{Hg}$-moderate $\mathrm{PAH}$; and more than $60 \mathrm{~mm}$ 
Hg-severe PAH. Left ventricular ejection fraction (LVEF), right ventricular dilatation, valvular abnormality, and pericardial effusion were documented.

- Antibody assay: Antinuclear antibody (ANA) for all patients was assessed using the immunofluorescence method-pattern (speckled, homogenous, cytoplasmic) and titer were measured. Anticardiolipin (ACL) antibody test (both immunoglobulin G [IgG] and immunoglobulin M

Table 1 Baseline characteristics of the study patients $(n=71)$

\begin{tabular}{|c|c|c|}
\hline Variables & Frequency $(n=71)$ & Percentage (\%) \\
\hline Age (mean, SD) & 27.86 (9.89) & \\
\hline $\operatorname{Sex}(F / M)$ & $66 / 5$ & \\
\hline Mean RVSP (SD) & $\begin{array}{l}58.07 \mathrm{~mm} \mathrm{Hg} \\
(18.16)\end{array}$ & \\
\hline $\begin{array}{l}\text { Mean duration of } \\
\text { SLE (months) }\end{array}$ & 35.3 & \\
\hline $\begin{array}{l}\text { Mean duration } \\
\text { between SLE and } \\
\text { diagnosis of PAH } \\
\text { (months) }\end{array}$ & 26.4 & \\
\hline Low C3/C4 & 53 & 74.6 \\
\hline Sm-RNP positive & 37 & 52.1 \\
\hline Sm positive & 21 & 29.6 \\
\hline SSA positive & 26 & 36.6 \\
\hline Ro-52 positive & 25 & 35.2 \\
\hline SSB positive & 6 & 8.4 \\
\hline PCNA positive & 2 & 2.8 \\
\hline $\begin{array}{l}\text { Nucleosome } \\
\text { positive }\end{array}$ & 19 & 26.8 \\
\hline $\begin{array}{l}\text { Ribosomal P protein } \\
\text { positive }\end{array}$ & 22 & 30.9 \\
\hline Histone positive & 17 & 23.9 \\
\hline dsDNA positive & 15 & 21.1 \\
\hline $\mathrm{aCL}$ positive & 18 & 25.3 \\
\hline Serositis & 38 & 53.5 \\
\hline Vasculitis & 15 & 21.1 \\
\hline Skin thickening & 9 & 12.7 \\
\hline Nephritis & 44 & 61.9 \\
\hline $\begin{array}{l}\text { Raynaud } \\
\text { phenomenon }\end{array}$ & 11 & 15.5 \\
\hline
\end{tabular}

Abbreviations: F, female; $\mathrm{M}$, male; $\mathrm{PAH}$, pulmonary arterial hypertension; RVSP, right ventricular systolic pressure; SD, standard deviation; SLE, systemic lupus erythematous; Sm-RNP, Smith-Ribonucleoprotein.
[IgM]) was done for all patients using the enzyme-linked immunosorbent assay (ELISA) technique. ACL IgG $>11$ GPL and IgM $>15$ MPL were considered as positive. ANA immunoblot (EUROLINE test kit) provided in vitro determination of IgG class's human autoantibodies in serum against 12 different antigens-Sm-RNP, Smith, SS-A, SS-B, dsDNA, nucleosome, histone, a ribosomal P protein, PCNA, PM-Scl, Jo-cyt1, and AMA-M2. Euroimmune recommends interpreting results based on signal intensity. Signal intensity of more than 25 (>25) in the EURO-Line Scan Flatbed scanner generated a medium to the strong band and was considered a positive result for the particular antibody. Serum complement level was assessed using the immunoturbidimetry method, and $\mathrm{C} 3<90 \mathrm{mg} / \mathrm{dL}$ and $\mathrm{C} 4<10 \mathrm{mg} / \mathrm{dL}$ were considered hypocomplementemia.

\section{Statistical Analysis}

K-means cluster analysis (nonhierarchical clustering or quick cluster; SPSS version 10 software [BM SPSS Statistics 21 License Authorization Wizard]) was used to identify clustering in SLE-PAH patients based on autoantibody. Briefly, this first involved defining a disease metric to quantify the degree of similarity between autoantibody patterns in two patients. Euclidian distance (the square root of the sums of squared differences between patients for each autoantibody) was used. The clusters' initial centers were chosen in the first pass of data, and patients were assigned to the closest center. Next, the cluster centers were recalculated on the patients in the cluster. The patient process continued till the clusters' means did not shift more than a given cutoff value or until the iteration limit was reached. Because it was not known in advance how many autoantibody clusters would be observed, 3, 4, and then 5 clusters were specified, respectively, in the K-means analysis. The outputs from the analyses with 3,4 , and 5 clusters of patients were then compared with each other regarding the prevalence of the individual autoantibody not shifting more than a given cutoff value or until the iteration limit is reached. Finally, by clustering antibodies into 3 clusters, three distinct groups of patients with very different autoantibody profiles were obtained. After clustering, associations between final clusters and patient characteristics were assessed by one-way analysis of variance (ANOVA) with post hoc analysis for continuous variables. Fisher's exact Hamilton test for categorical variables was done; $p$-value < 0.05 was considered significant. All statistical analyses were performed using SPSS for Windows version 10.0.

Table 2 Description of the clusters

\begin{tabular}{|l|l|l|l|}
\hline Variable & Cluster 1 & Cluster 2 & Cluster 3 \\
\hline Antibodies & Sm-RNP, Smith, SSA & No definite antibody association & $\begin{array}{l}\text { dsDNA, nucleosome, histone, } \\
\text { ribosomal P protein }\end{array}$ \\
\hline Low complements & Yes & Yes & Yes \\
\hline Anticardiolipin, anti-La & No cluster & No cluster & No cluster \\
\hline Total cases & 26 & 36 & 9 \\
\hline
\end{tabular}




\section{Results}

\section{A. Baseline Characteristics}

A total of 71 patients with pulmonary hypertension were identified and included in the analysis. The demographic characteristics, the frequency of specific autoantibodies, and various clinical phenotypes are shown in - Table 1; $12.7 \%$ of the patients had associated skin thickening and $15.5 \%$ of patients were found to have the Raynaud phenomenon.

\section{B. Clustering of Patients}

Three distinctive antibody clusters were identified-cluster 1 (anti-Sm-RNP, anti-Sm, anti-SSA), cluster 2 (no definitive autoantibody association), and cluster 3 (anti-dsDNA, nucleosome, histone, ribosomal $\mathrm{P}$ protein). Twenty-six patients were assigned to cluster 1,36 patients were assigned to cluster 2, and 9 patients were assigned to cluster 3. Low complements were found in all three clusters, while anticardiolipin and anti-SSB did not show specific association with any cluster. In cluster 2 , the anticardiolipin antibody was present in 10 patients, which was not statistically significant. - Table 2 depicts the three clusters with their antibody association. The frequency of the specific autoantibodies in each cluster is shown in -Table 3.

\section{Demographic and Clinical Characteristics across Clusters}

The demographic characteristics and clinical manifestations of SLE patients in the three clusters is shown in - Table 4. A significant association with cytopenia was seen in cluster 2 (77.8\%) as compared to clusters 1 (42.3\%) and 3 (55.6\%) ( $p$ 0.01, Fisher value 8.25). Cluster 3 patients had significantly higher disease activity ( $p 0.049$, Fisher value 5.93 ) and dsDNA association ( $p$ 0.001, Fisher 14.33) (-Table 4).

\section{Mean RVSP in Different Clusters}

Mean ( \pm standard deviation [SD]) RVSP in clusters 1,2 , and 3 were $52.9 \pm 15.9,64.7 \pm 18.6$, and $46 \pm 11.4 \mathrm{~mm} \mathrm{Hg}$, respectively ( - Table 5) with significant high value in cluster 2 ( $p$ 0.003) (by ANOVA testing). Post hoc analysis showed that cluster 2 had significantly higher mean RVSP than clusters 1 and 3 ( $p 0.023$ and 0.011 ) (-Table 6).

\section{Discussion}

In this study, using clustering techniques for autoantibodies, three clusters were identified. The largest cluster of patients had no definite antibody association. The next large cluster was associated with anti-Sm-RNP, anti-Smith, and anti-Ro antibodies, while the smallest cluster included anti-dsDNA, antinucleosome, antihistone, and antiribosomal $\mathrm{P}$ protein antibodies. In patients with no definite autoantibodies, significantly more frequent cytopenia, skin thickening, and higher RVSP were noted.

There are two published studies on clustering analysis in lupus patients with associated PAH. Sun et al $^{6}$ used derivation and validation cohorts, each having approximately 100 patients of SLE-PAH. They reported that patients clustered into high and low disease activity groups, with the former having less time to diagnose. They adjusted for immunosuppressive and vasodilator treatments and noted that survival differences existed between the two groups. This study did not use antibodies as independent variables.

Mizus $^{1}$ studied 10 serological variables in 154 patients with SLE-PAH. One of their five clusters was defined by antiphospholipid antibodies and another by anti-Ro and anti-La. The third, as in our study, had a low frequency of all antibodies. Of note, this work was derived from the Hopkins Lupus Cohort. Though the numbers in our study are slightly less than this study, the composition of antibodies in our cluster is different from that in the Hopkins Lupus Cohort. Anticardiolipin antibodies did not figure in any cluster in our study. The cluster with significant numbers and definite antibody representation included anti-Smith and anti-Sm-RNP. This difference may highlight the genetic differences which shape autoantibody specificity among Indian and Hopkins Lupus Cohort of SLE-PAH.

There have been many cluster analysis publications in 2005, 2012, 2015, and 2019 on SLE patients. The first publication ${ }^{7}$ of cluster analysis done on 1,257 patients tested on seven autoantibodies identified three clusters with an almost

Table 3 Frequency of specific autoantibodies in various clusters

\begin{tabular}{|l|l|l|l|}
\hline Antibody & Cluster1 (\%) & Cluster 2 (\%) & Cluster 3 (\%) \\
\hline Anti-Sm-RNP & $25(96.2)$ & $9(25)$ & $3(33.3)$ \\
\hline Anti-Sm & $18(69.2)$ & $0(0)$ & $3(33.3)$ \\
\hline Anti-SSA & $13(50)$ & $11(30.6)$ & $2(22.2)$ \\
\hline Anti-dsDNA & $5(19.2)$ & $3(8.3)$ & $7(77.8)$ \\
\hline Antiribosomal P & $9(34.6)$ & $8(22.2)$ & $5(55.6)$ \\
\hline Antinucleosome & $10(38.5)$ & $0(0)$ & $9(100)$ \\
\hline Antihistone & $6(23.1)$ & $2(5.6)$ & $9(100)$ \\
\hline Complement & $25(96.2)$ & $21(58.3)$ & $7(77.8)$ \\
\hline Anticardiolipin & $5(19.2)$ & $10(27.8)$ & $3(33.3)$ \\
\hline
\end{tabular}


Table 4 Comparison of demographic and clinical features according to antibody cluster

\begin{tabular}{|c|c|c|c|c|c|}
\hline Variables & $\begin{array}{l}\text { Cluster } 1 \\
\text { (percentage) }\end{array}$ & $\begin{array}{l}\text { Cluster } 2 \\
\text { (percentage) }\end{array}$ & $\begin{array}{l}\text { Cluster } 3 \\
\text { (percentage) }\end{array}$ & $\begin{array}{l}\text { Fisher-Hamilton } \\
\text { exact value }\end{array}$ & $p$-Value \\
\hline Number of patients & 26 & 36 & 09 & & \\
\hline Age (mean \pm SD) & $25.96( \pm 8.8)$ & $30.06( \pm 10.74)$ & $24.56( \pm 8.02)$ & & \\
\hline $\operatorname{Sex}(F / M)$ & $24 / 2$ & $33 / 3$ & All female & 0.437 & 1.0 \\
\hline Skin thickening & $1(3.8)$ & $8(22.2)$ & $1(11.1)$ & 4.13 & 0.099 \\
\hline $\mathrm{RP}$ & $2(7.7)$ & $7(19.4)$ & $2(22.2)$ & 2.15 & 0.38 \\
\hline Vasculitis & $8(30.7)$ & $5(13.9)$ & $2(22.2)$ & 2.66 & 0.31 \\
\hline Myositis & $5(19.2)$ & $4(11.1)$ & $1(11.1)$ & 0.94 & 0.72 \\
\hline MNM & 0 & $2(5.6)$ & 0 & 1.49 & 0.62 \\
\hline CVA & $1(3.8)$ & $6(16.7)$ & 0 & 2.93 & 0.18 \\
\hline Nephritis & $17(65.4)$ & $20(55.6)$ & $7(77.8)$ & 1.59 & 0.46 \\
\hline Serositis & $15(57.7)$ & $18(50)$ & $5(55.6)$ & 0.44 & 0.85 \\
\hline ILD & $2(7.7)$ & 5 (13.9) & $1(11.1)$ & 0.69 & 0.87 \\
\hline Pregnancy morbidity & $5(19.2)$ & $7(19.4)$ & 0 & 1.77 & 0.49 \\
\hline Cytopenia & $11(42.3)$ & $28(77.8)$ & $5(55.6)$ & 8.25 & 0.01 \\
\hline $\begin{array}{l}\text { Disease activity (SLEDAI } \\
\text { based) }\end{array}$ & $\begin{array}{l}\text { 20-HDS, } \\
6 \text {-LDS }\end{array}$ & $\begin{array}{l}19-\mathrm{HDS} \\
17-\mathrm{LDS}\end{array}$ & $\begin{array}{l}\text { 8-HDS, } \\
\text { 1-LDS }\end{array}$ & 5.93 & 0.049 \\
\hline dsDNA positivity & $17(65.4)$ & $13(36.1)$ & $9(100)$ & 14.33 & 0.001 \\
\hline aCL positivity & $5(19.2)$ & $10(27.8)$ & $3(33.3)$ & 1.071 & 0.599 \\
\hline CYC & $10(38.5)$ & $16(44.4)$ & $5(55.6)$ & 0.86 & 0.67 \\
\hline
\end{tabular}

Abbreviations: CVA, cerebro-vascular accident; CYC, cyclophosphamide; dsDNA, double stranded DNA; F, female; ILD, interstitial lung disease; M, male; MNM, mononeuritis multiplex; RP, Raynaud phenomenon; SD, standard deviation; SLEDAI, SLE disease activity index.

Table 5 Mean RVSP in clusters

\begin{tabular}{|l|l|l|l|l|l|}
\hline Cluster & N & Mean & \multirow{2}{*}{ SD } & \multicolumn{2}{|c|}{ 95\% Confidence interval for mean } \\
\cline { 3 - 6 } & & RVSP & & Lower bound & Upper bound \\
\hline 1 & 26 & 52.96 & 15.94 & 46.52 & 59.40 \\
\hline 3 & 36 & 64.78 & 18.59 & 58.49 & 71.07 \\
\hline Total & 71 & 46.0 & 11.37 & 37.26 & 54.74 \\
\hline
\end{tabular}

Abbreviation: RVSP, right ventricular systolic pressure.

Table 6 ANOVA with post hoc analysis for RVSP in between clusters

\begin{tabular}{|c|l|l|l|l|l|l|}
\hline \multirow{2}{*}{$(\mathrm{I})$ cluster } & (J) cluster & $\begin{array}{l}\text { Mean } \\
\text { difference (I-J) }\end{array}$ & $\begin{array}{l}\text { Standard } \\
\text { error }\end{array}$ & \multirow{2}{*}{$p$-value } & \multicolumn{2}{|c|}{ 95\% Confidence interval } \\
\cline { 3 - 7 } & & & & & Lower bound & Upper bound \\
\hline \multirow{2}{*}{1} & 2 & -11.82 & 4.36 & .023 & -22.26 & -1.38 \\
\cline { 2 - 7 } & 3 & 6.96 & 6.55 & .540 & -8.72 & 22.65 \\
\hline \multirow{2}{*}{3} & 1 & 11.82 & 4.36 & $\mathbf{. 0 2 3}$ & 1.38 & 22.26 \\
\cline { 2 - 7 } & 3 & 18.78 & 6.31 & $\mathbf{. 0 1 1}$ & 3.66 & 33.89 \\
\hline
\end{tabular}

Abbreviations: ANOVA, analysis of variance; RVSP, right ventricular systolic pressure.

similar number of patients in the groups. The cluster with dsDNA, aCL, and LAC had the highest incidence of thrombosis and stroke damage. The cluster, which included anti-Ro, anti-La antibodies, had the highest presence of Sjogren syndrome. The cluster with the Sm-RNP antibody had the lowest frequency of cytopenia. $\mathrm{Li}^{8}$ from Hongkong reported on 1,928 patients, identified the dsDNA cluster with nephritis; the Sm-RNP, Sm, aCL cluster with arthritis and serositis; and the anti-Ro/anti-La cluster with photosensitivity. In a Chinese cohort ${ }^{9}$ of 917 patients, three clusters including no 
ENA were identified, and differences in age of onset of lupus and other clinical features were reported. Another cluster analysis published from Johns Hopkins cohort analyzing only seven autoantigens, including Ro and RNP with different molecular weights, found two clusters. The Sm cluster was associated with serositis. From Turkey, ${ }^{10} 852$ lupus patients clustered into five clusters, one that did not have a high frequency of any antibodies but a high organ involvement. Their Sm-RNP cluster is associated with Raynaud's phenomenon and $\mathrm{PAH}$.

The concept of two different pathophysiological mechanisms of SLE-PAH in groups with different disease activities, as proposed by Sun et al, can be extrapolated to suggest the possibility of different pathological mechanisms at play in the cluster which lacked a definite antibody association in our study.

The use of immunosuppressive therapy has been reported to predict short-term survival in CTD-PAH. ${ }^{11}$ In an open-labeled observational study, we have also previously reported that immunosuppressant therapy may have a modest benefit in improving PAH in SLE patients. ${ }^{12}$ Hence at the bedside, the choice of whether to use potentially toxic immunosuppressive therapy in SLE-PAH is guided by active lupus elsewhere and serological activity in a patient. Any further study would need to account for these confounders while analyzing autoantibodies as independent predictors of survival in SLE-PAH patients.

Our study's limitations were that pulmonary artery pressure was not documented by right heart catheterization, and other antiphospholipid antibodies (LAC, anti-B2 glycoprotein 1) were not measured.

We hope to repeat this exercise on a larger SLE-PAH and SLE group to reproduce the ethnic variation in autoantibodies in lupus and its subgroups. We are surprised to report that patients with Sm-RNP did not have a higher incidence of skin thickening or Raynaud's phenomenon, suggesting underlying scleroderma as a PAH cause. This reiterates that the patients included in our study have no underlying scleroderma and constitute an SLE-PAH population.

\section{Conclusion}

This study confirms that in SLE-PAH, autoantibodies do exist as clusters, and this association has a bearing on phenotypic manifestations. The largest cluster had no definite antibody association but the most severe PAH. The underlying differences in different pathophysiologic autoantibody clusters will need further studies.

\section{Audio}

Audio file for this article is available at https://doi. org/10.1055/s-0041-1732510.

\section{Conflict of Interest}

None declared.

\section{References}

1 Mizus M, Li J, Goldman D, Petri MA. Autoantibody clustering of lupus-associated pulmonary hypertension. Lupus Sci Med 2019;6(1):e000356

2 Li M, Wang Q, Zhao J, et al. CSTAR co-authors. Chinese SLE Treatment and Research group (CSTAR) registry: II. Prevalence and risk factors of pulmonary arterial hypertension in Chinese patients with systemic lupus erythematosus. Lupus 2014;23(10):1085-1091

3 Lian F, Chen D, Wang Y, et al. Clinical features and independent predictors of pulmonary arterial hypertension in systemic lupus erythematosus. Rheumatol Int 2012;32(6):1727-1731

4 Prabu A, Patel K, Yee C-S, et al. Prevalence and risk factors for pulmonary arterial hypertension in patients with lupus. Rheumatology (Oxford 2009;48(12):1506-1511

5 Hachulla E, Jais X, Cinquetti G, et al. French Collaborators Recruiting Members $\left({ }^{*}\right)$. Pulmonary arterial hypertension associated with systemic lupus erythematosus: results from the French pulmonary hypertension registry. Chest 2018;153(1):143-151

6 Sun F, Lei Y, Wu W, et al. Two distinct clinical phenotypes of pulmonary arterial hypertension secondary to systemic lupus erythematosus. Ann Rheum Dis 2019;78(1):148-150

7 To $\mathrm{CH}$, Petri M. Is antibody clustering predictive of clinical subsets and damage in systemic lupus erythematosus. ? Arthritis Rheum 2005;52(12):4003-4010

8 Li PH, Wong WHS, Lee TL, et al. Relationship between autoantibody clustering and clinical subsets in SLE: cluster and association analyses in Hong Kong Chinese. Rheumatology (Oxford 2013;52(2):337-345

9 Tang X, Huang Y, Deng W, Tang L, Weng W, Zhang X. Clinical and serologic correlations and autoantibody clusters in systemic lupus erythematosus: a retrospective review of 917 patients in South China. Medicine (Baltimore 2010;89(1):62-67

10 Artim-Esen B, Çene E, Şahinkaya Y, et al. Cluster analysis of autoantibodies in 852 patients with systemic lupus erythematosus from a single center. J Rheumatol 2014;41(7):1304-1310

11 Yasuoka H, Shirai Y, Tamura Y, Takeuchi T, Kuwana M. Predictors of favorable responses to immunosuppressive treatment in pulmonary arterial hypertension associated with connective tissue disease. Circ J 2018;82(2):546-554

12 Kommireddy S, Bhyravavajhala S, Kurimeti K, et al. Pulmonary arterial hypertension in systemic lupus erythematosus may benefit by addition of immunosuppression to vasodilator therapy: an observational study. Rheumatology (Oxford 2015;54(9):1673-1679 